\title{
Regulation of ubiquitination and degradation of CRTC1 transcriptional coactivator by salt-inducible kinase SIK1 \\ Gao Wei Wei (HKU)
}

(Supervisor: Professor DY Jin, HKU)

cAMP response element (CRE) binding protein (CREB) is a bZIP transcription factor centrally involved in the regulation of cellular proliferation, survival, energy metabolism and stress response. CREB-regulated transcriptional coactivator (CRTC) is an essential factor which potently activates the transcriptional activity of CREB by promoting the recruitment of TAFII130/135. Currently, several kinases and phosphatases are known to regulate the function of CRTCs. For example, salt-inducible kinases (SIKs) belonging to AMPK protein kinase family inhibit the activity of CRTCs through a phosphorylation-dependent nuclear export mechanism. In this study, we provide the first evidence for destabilization of CRTC1 protein by SIK1. Enforced expression of SIK1 resulted in diminution of the steady-state amount of CRTC1, whereas expression of kinase-defective mutant of SIK1 had no influence on the level of CRTC1, indicating the requirement of the kinase activity of SIK1 for the suppressive effect on CRTC1. Addition of proteasome inhibitor MG132 restored CRTC1 protein level. More importantly, ubiquitinated CRTC1 species were detected in the ubiquitin pull down assay in the presence of kinase-proficient SIK1. Taken together, our data suggest a novel regulatory mechanism for CRTC1 through which SIK1 suppresses the CREB-mediated gene transcription by destabilizing CRTC1 in a phosphorylation-induced, ubiquitination-mediated and proteasome-dependent manner.

\section{Role of ATM Signaling in PALB2-dependent DNA Damage Responses}

Yingying Guo ${ }^{* 1,2,3}$, Wanjuan Feng ${ }^{1,2,3}$, Shirley M.H. Sy ${ }^{4} \&$ Michael S.Y. Huen ${ }^{1,2,3}$

${ }^{1}$ Genome Stability Research Laboratory, ${ }^{2}$ Department of Anatomy, ${ }^{3}$ Center for Cancer Research,

${ }^{4}$ Department of Biochemistry, The University of Hong Kong

Mutations of the PALB2 gene lead to a number of hereditary cancer-predisposing syndromes, including Fanconi anemia and hereditary breast and ovarian cancer syndrome. Originally identified as a core DNA repair factor, emerging evidence now implicates PALB2 in cell cycle checkpoint control, DNA replication, oxidative stress regulation and transcription, highlighting the multi-functionality of the tumor suppressor. Notably, mechanistically how its expanding repertoire of functions are orchestrated remains unexplored.

By halting cell cycle progression, checkpoint machineries allow time for restoring of genome integrity prior to resumption of cell growth and division. However, it is not well understood how cell cycle arrest is coordinated with DNA repair processes, and whether DNA repair proteins signal to restart cell cycle following completion of DNA repair. Intriguingly, recent observations that PALB2 plays a role in cell cycle regulation raise the interesting possibility that the tumor suppressor may encode a protein hub to coordinate DNA repair and cell proliferation. However, exactly how PALB2 function in cell cycle checkpoints may be spatiotemporally coupled to DNA repair is not known.

In exploring how DNA damage signaling coordinates with PALB2-dependent DNA repair, we have identified a panel of putative phosphorylation sites on the PALB2 polypeptide. Employing a combination of biophysical, biochemical and genetic approaches, we found that ionizing radiation (IR) triggered PALB2 phosphorylation at Ser157 and Ser376 in an ataxia telangiectasia mutated (ATM) kinase-dependent manner. Interestingly, our data indicated that PALB2 phosphorylation status at Ser157 and Ser376 neither affected its relocalisation to DNA damage sites nor were they required for DNA repair. Instead, these DNA damage-regulated PALB2 phosphorylation events are temporally coupled to cell recovery from checkpoint arrest. We propose that ATM-mediated PALB2 phosphorylation represents a means to coordinate cell cycle checkpoint regulation and DNA repair processes. Given the potential relationship between PALB2 phosphorylation and tumorigenesis, our study may provide a diagnostic biomarker of cancer predisposition. 